\title{
Violência Sexual contra Mulheres: um Estudo Comparativo entre Vítimas Adolescentes e Adultas
}

\author{
Mykaella Cristina Antunes Nunes \\ Rebeca Fernandes Ferreira Lima \\ Universidade de Fortaleza, CE, Brasil \\ Universidade de Fortaleza, CE, Brasil \\ Normanda Araujo de Morais \\ Universidade de Fortaleza, CE, Brasil
}

\begin{abstract}
Resumo: Este estudo teve como objetivo descrever as características da vítima, da violência, do agressor e do atendimento recebido por mulheres (12 anos acima) em um hospital da rede pública de Fortaleza-CE entre 2010 e 2013. Buscou-se, ainda, identificar as consequências da violência e o desfecho da gestação (continuidade ou interrupção), além de realizar uma análise comparativa das vítimas adolescentes e adultas. Elaborou-se um formulário específico de investigação (36 questões), preenchido com base em três formulários utilizados no hospital. No total, foram analisados 112 formulários, dos quais 51 pertenciam a adolescentes e 61 a adultas. Os dados foram analisados por meio de estatísticas descritivas e inferenciais no Statistical Package for Social Science - SPSS, versão 19. As vítimas eram em sua maioria adultas, de raça/cor parda, heterossexual, solteira e estudante. A violência sexual mais praticada foi o estupro e por agressor desconhecido por parte das vítimas. A gravidez foi a consequência da violência sexual mais frequente, tendo as mulheres adolescentes mais vezes optado pela continuidade da gestação. A evolução do atendimento não ficou clara em muitos casos, devido à fragilidade de preenchimento das fichas analisadas. Ressalta-se a importância do estudo que, ao incluir diversas perspectivas acerca da violência sexual (vítima, violência, agressor e atendimento), pode contribuir para a elaboração e estruturação de políticas públicas.
\end{abstract}

Palavras-chave: Violência Sexual, Gravidez Indesejada, Assistência Hospitalar. 


\title{
Sexual Violence against Women: a Comparative Study between Adolescent and Adult Victims
}

\begin{abstract}
This study aimed to describe the characteristics of the victim, the violence, the aggressor and the care received by women (12 years old and above) in a public hospital in the city of Fortaleza-CE between 2010-2013. The study also sought to identify the consequences of violence and the outcome of the pregnancy (continuity or interruption), and perform a comparative analysis of adolescent and adult victims. An specific form of research (36 questions) was elaborated and filled out based on three forms used at the hospital. A total of 112 forms were analyzed, from which 51 belonged to adolescents and 61 to adults. The data were analyzed using descriptive and inferential statistics in the Statistical Package for Social Science - SPSS (version 19). The victims were in their majority adult of mixed race / color, heterosexual, single and student. The most practiced sexual violence was rape and by an attacker unknown to the victims. The pregnancy was the result of more recurrent sexual violence, and adolescent women more often opted for the continuation of the pregnancy. The evolution of care is not clear in many cases, due to the inconsistence on the filling out of the analyzed forms. The importance of the study, which includes several perspectives on the SV (victim, violence, abuser, and service), can contribute to the development and structuring of public policies.
\end{abstract}

Keywords: Sexual Violence, Unwanted Pregnancy, Hospital Care.

\section{Violencia Sexual contra mujeres: un Estudio Comparativo entre Víctimas Adolescentes y Adultas}

Resumen: Este estudio tuvo como objetivo describir las características de la víctima, la violencia, el agresor y la atención que reciben las mujeres (mayores de 12 años) en un hospital público de Fortaleza-CE entre 2010-2013. Se buscó también para identificar las consecuencias de la violencia y el resultado del embarazo (continuidad o interrupción), y realizar un análisis comparativo de las víctimas adolescentes y adultos. Se elaboró un Informe Específico de Investigación (36 preguntas), rellenado con base en tres formularios usados en el hospital. En total, 112 formas analizadas, de las cuales 51 pertenecían a adolescentes y 61 a adultos. Los dados se analizaron mediante estadística descriptiva y con inferencias en el Statistical Package for Social Science - SPSS (versión 19). Las víctimas eran en su mayoría adultas de raza mixta/color pardo, heterosexuales, solteras y estudiantes. La violencia sexual más practicada fue el estupro y por agresor desconocido por parte de las víctimas. El embarazo fue el resultado de la violencia sexual más frecuente, y las mujeres adolescentes muy a menudo optaron por la continuación de la gestación. La evolución del atendimiento no quedó clara en muchos casos, debido a la fragilidad del relleno de los formularios analizados. Se resalta la importancia del estudio, que al incluir diversas perspectivas sobre de la VS (víctima, violencia, abusador, y atendimiento), puede contribuir a la elaboración y estructuración de políticas públicas.

Palabras clave: Violencia Sexual, Embarazo no Deseado, Atención Hospitalaria. 


\section{Introdução}

No Relatório Mundial sobre Violência e Saúde (World Health Organization, 2002), a violência sexual (VS) é definida a partir de suas múltiplas formas de apresentação, sendo: qualquer ato sexual, tentativa de obter um ato sexual, comentários ou investidas sexuais indesejadas, ou atos direcionados ao tráfico sexual. Além disso, a VS volta-se contra a sexualidade de uma pessoa, por meio da coação praticada por qualquer pessoa, independentemente de sua relação com a vítima e em qualquer cenário, inclusive em casa e no trabalho, mas não limitado a eles.

No Brasil, o estupro é definido juridicamente como sendo o ato de "constranger alguém, mediante violência ou grave ameaça, a ter conjunção carnal ou a praticar ou permitir que com ele se pratique outro ato libidinoso" (Brasil, 2012, p. 27), podendo ser cometido contra mulheres e homens, a partir de reformulação da Lei no 12.015 de 2009 (Brasil, 2009). Assim, atos sexualmente violentos podem ocorrer contra pessoas de ambos os sexos e de diversas faixas etárias. No entanto, a literatura tem apresentado as mulheres como as principais vítimas (Cerqueira, \& Coelho, 2014).

Constatou-se ainda a prevalência de mulheres da faixa etária infantojuvenil (Veloso, Magalhães, Dell'Aglio, Cabral, \& Gomes, 2013) e jovens adultas (Facuri, Fernandes, Oliveira, Andrade, \& Azevedo, 2013) vítimas da VS. Entre as razões para tal fato estão a condição de fragilidade das mulheres em relação aos homens, seja pela sujeição ao poder exercido pelo mais velho sobre o mais novo ou pela assimetria de gênero (masculino/feminino) (Faúndes, Rosas, Bedone, \& Orozco, 2006; Gomes, Falbo Neto, Viana, \& Silva, 2006; Lima, \& Deslandes, 2014; Machado, Fernandes, Osis, \& Makuch, 2015). Tais aspectos dizem respeito a uma construção histórica e social de opressão das mulheres em relação aos homens, que opera há séculos em espacialidades e temporalidades distintas de realidade e condição humanas e que, por tais motivos, as tornam mais vulneráveis aos homens na esfera sexual (Matos, 2008).

As consequências dessa violência para as vítimas são inúmeras. Mulheres que sofreram VS estão mais propensas ao desenvolvimento de sintomas psiquiátricos como transtorno de estresse pós-traumático (TEPT), depressão, somatizações, tentativas de suicídio e uso de substâncias psicoativas. Além de estarem expostas a contrair doenças sexualmente transmissíveis (DST) e a terem gravidez indesejada (Machado et al., 2015; Nunes, \& Morais, 2016). No que se refere às mulheres grávidas decorrente do estupro, verificaram-se casos com criança (Santos, 2012), com adolescentes (Cantelmo, Cavalcante, \& Costa, 2011; González, Luengo, Caba, \& Molina, 1999; Moura, Silveira, \& Nogueira, 2001) e com mulheres adultas (Drezett et al., 2011; Vertamatti et al., 2009), confirmando-se a existência do problema da VS e gravidez nas diversas faixas etárias.

Com o fim de proteger e assegurar um atendimento de qualidade às vítimas de VS, desde 1998, o Ministério da Saúde do Brasil padronizou o atendimento multidisciplinar para as vítimas de VS por meio da Norma Técnica. O atendimento emergencial, nas primeiras 72 horas após a violência, tem por objetivo acolhimento e administração de anticoncepção de emergência e a profilaxia para as DSTs, doenças virais e bacterianas. A Norma Técnica também prevê atendimento às mulheres que solicitam interrupção legal da gestação nos casos de gravidez decorrente de estupro, situação prevista no Código Penal Brasileiro desde 1940 (Brasil, 2012).

Nesse contexto, portanto, entende-se como relevante a realização de estudos que busquem descrever as características das vítimas, da violência sofrida e suas consequências, assim como sobre o suposto agressor (geralmente menos enfocado nos estudos) e o atendimento que a vítima tem ao buscar ajuda na rede hospitalar. Igualmente importante é verificar especificidades das diferentes faixas etárias quanto às características da VS sofrida pelas vítimas como: contexto intra ou extrafamiliar; vínculo com o agressor (pessoa íntima/conhecida ou desconhecida); rede de apoio informal disponível (família, amigos, comunidade etc.); desenvolvimento físico e emocional, entre outros aspectos que diferenciam a vivência entre as vítimas.

A descrição desses múltiplos aspectos pode auxiliar na estruturação e qualificação de modelos de atendimento; ao passo que a falta de informação pode prejudicar o conhecimento mais detalhado do fenômeno, interferindo nas ações de planejamento, organização e operacionalização dos serviços de saúde (Facuri et al., 2013). O atendimento psicológico é imprescindível nesta área de atuação, dada as consequências negativas que a VS pode gerar. Tão essencial quanto a assistência psicológica são os outros atendimentos realizados às vítimas pelos demais 
profissionais do serviço hospitalar (por exemplo, profilaxias de emergência - a anticoncepção de emergência, a prevenção contra DST/AIDS e hepatite B), que devem estar atentos à multiplicidade de variáveis que envolvem uma situação de VS.

Deste modo, este estudo teve como objetivo descrever as características da vítima, da violência, do agressor e do atendimento recebido por mulheres (12 anos acima) em um hospital da rede pública de Fortaleza-CE entre 2010 e 2013. Buscou-se, ainda, identificar as consequências da violência e o desfecho da gestação (continuidade ou interrupção), além de realizar uma análise comparativa das vítimas adolescentes e adultas. Espera-se que esse estudo possa se somar ao campo de conhecimentos e intervenções sobre VS, ao permitir uma análise da mesma a partir de múltiplos ângulos (vítima, violência, agressor, atendimento), bem como pelo seu diferencial em enfocar a gravidez e o desfecho da mesma (interrupção ou continuidade) a partir de uma comparação entre dois grupos etários distintos - adolescentes e adultas.

\section{Método}

\section{Delineamento}

Consiste num estudo quantitativo, exploratório e descritivo, de caráter retrospectivo, realizado através da análise documental a respeito dos casos de violência sexual contra mulheres adolescentes (entre $12 \mathrm{e}$ 18 anos de idade, segundo o Estatuto da Criança e do Adolescente [Brasil, 1990]) e adultas, atendidas em um hospital público da cidade de Fortaleza-CE durante o período 2010-2013.

\section{Participantes}

Foram incluídos neste estudo formulários referentes a 112 mulheres vítimas de violência sexual, sendo 51 adolescentes e 61 adultas. Esses formulários foram selecionados de um total de 156 fichas, inicialmente encontradas no hospital, referente ao período 2010-2013. Foram incluídas as fichas de participantes que atendiam aos seguintes critérios de inclusão: vítimas com idade igual ou superior a 18 anos; que tenham sofrido violência sexual (somada ou não a outros tipos de violência) e cuja violência sexual estava explicitada na sua ficha de notificação. Dessa forma, foram excluídos da análise os casos de violência sexual contra criança $(n=25)$; os casos que somente diziam respeito à violência física, psicológica/moral ou outro tipo de violência diferente da sexual ( $\mathrm{n}=16)$; e os casos que não apresentavam a tipologia da violência $(n=3)$.

\section{Instrumento}

Utilizou-se um formulário específico elaborado especialmente para esse estudo, com base nas Fichas de Notificação/Investigação Individual, de Dados Sociais e de Avaliação Psicológica utilizadas no hospital em questão. O formulário continha 36 questões, as quais diziam respeito aos seguintes aspectos: dados sociodemográficos da vítima (idade, sexo, raça/cor, religião, escolaridade, ocupação etc.); características da violência sexual (local de ocorrência, meio de agressão, tipo de violência sexual, práticas sexuais realizadas etc.); dados sobre o suposto agressor (número de envolvidos, grau de ligação com a vítima, sexo do provável autor da agressão etc.); consequências da violência sexual (estresse pós-traumático, DSTs, gravidez etc.); dados acerca do atendimento hospitalar e de outros serviços (notificação, encaminhamentos etc.); e desfecho da gestação.

\section{Procedimentos de coleta dos dados}

A coleta das informações foi realizada primeiramente a partir do levantamento de todos os casos de violência sexual que deram entrada no período 2010-2013 no hospital. Em seguida, para cada caso de violência sexual identificado, foram verificados os três modelos de fichas (Notificação/Investigação Individual, de Dados Sociais e de Avaliação Psicológica), os quais serviram de base, por sua vez, para o preenchimento do formulário deste estudo. As fichas caracterizam-se por serem de autorrelato das vítimas. Dessa forma, os dados analisados que compuseram o formulário foram na percepção da vítima, mas preenchidos pelos profissionais. A primeira autora desse artigo, em parceria com uma estudante de graduação do curso de Psicologia, devidamente treinada para essa tarefa, foram as responsáveis pela sistematização dessas informações.

Destaque-se que a coleta de dados foi um processo árduo, pois se verificou ausência de fichas no local da investigação principal (Núcleo de Epidemiologia), tendo sido necessário localizar algumas fichas em outros setores do hospital. Feita a pesquisa em três setores (Núcleo de Epidemiologia, Psicologia e Serviço 
Social), identificou-se que havia casos que só podiam ser encontrados em um setor, constatando-se, portanto, a inexistência de alguns dados. Apesar dessa fragilidade, buscou-se ao máximo revertê-la, realizando a pesquisa em mais departamentos que o inicialmente estabelecido. Os locais de coleta se apresentaram salubres e sigilosos na medida do possível para a realização da pesquisa, não tendo sido um empecilho. Já quanto aos profissionais dos setores em que se buscou o material de coleta, obteve-se dificuldade de acesso aos instrumentais em um dos departamentos, que depois foi sanada contatando-se outro profissional. Ao final da coleta, os dados foram digitados no software Statistical Package for Social Science (SPSS), versão 19.

\section{Procedimentos de análise dos dados}

As análises foram realizadas através do SPSS e o nível de significância adotado foi de $p<0,05$. Foram calculadas as frequências, porcentagens, médias e desvios-padrão para as variáveis de descrição sociodemográfica das vítimas, caracterização da violência e do agressor, consequências da violência, atendimento e desfecho da gestação. Depois, os dados de caracterização da violência (número e tipologia), início do atendimento, quantidade de agressores, consequências da violência e desfecho da gestação foram analisados utilizando-se Teste $t$-Student para comparações entre adolescentes e adultas. Além disso, utilizou-se a Correlação de Pearson para verificar correlações entre essas variáveis.

Para as análises inferenciais foram criados os seguintes indicadores: número de violência-composto pela ocorrência das violências física, sexual, psicológica/moral, tortura, financeira e negligência; violência intrafamiliar -0 = Não e $1=$ Sim; início do atendimento $-0=$ dentro de $72 \mathrm{~h}, 1=1$ mês e $2=2$ meses ou mais; consequência/gravidez $-0=$ Não e $1=$ Sim; e desfecho da gestação $-1=$ continuidade e 2 = interrompeu.

\section{Procedimentos éticos}

Esse estudo recebeu aprovação do Comitê de Ética da Universidade a qual suas autoras estão filiadas (Parecer no 2310094836735), bem como do hospital onde foi realizado (Parecer $n^{\circ} 625.809$ ).

\section{Resultados}

A partir da análise dos formulários de investigação, delimitaram-se alguns resultados que foram organizados de acordo com as seguintes seções: descrição das vítimas; caracterização da violência e do agressor; e atendimento recebido, consequências da violência e desfecho da gestação.

\section{Descrição das vítimas}

Nos formulários analisados ( $\mathrm{n}=112)$ foram identificadas apenas vítimas do sexo feminino, na faixa etária entre 12 e 57 anos $(M=22$ anos; $D P=9,3)$, sendo $51(46,4 \%)$ adolescentes e $61(53,6 \%)$ adultas. Foi registrada uma maioria de raça/cor parda $(n=62 ; 55,4 \%)$, heterossexual ( $\mathrm{n}=72 ; 64,3 \%)$; solteira $(\mathrm{n}=81 ; 72,3 \%)$ e com religião, sendo 51 (45,5\%) católicas e $24(21,4 \%)$ evangélicas. Quanto à ocupação, verificou-se que 57 $(50,9 \%)$ eram estudantes, $38(37,5 \%)$ trabalhavam e $5(4,5 \%)$ estavam desempregadas. A maioria tinha o ensino fundamental incompleto $(n=32 ; 28,6 \%)$, apenas $10(8,9 \%)$ concluíram o ensino médio e três $(2,7 \%)$, o ensino superior. Entre as mulheres, 16 (19,8\%) tinham comprometimento de ordem física, psicológica, mental ou cognitiva.

\section{Caracterização da violência e do agressor}

Conforme os critérios de inclusão do estudo, todas as mulheres haviam sofrido VS. Conforme mostra a Tabela 1 , além da VS, a maioria $(\mathrm{n}=78 ; 75 \%)$ sofreu outros tipos de violência, que foram: física, psicológica/moral, tortura, financeira e negligência. Embora não se tenha verificado diferença significativa, observou-se uma tendência de as adolescentes relatarem mais de um tipo de violência (por exemplo, física, sexual e psicológica) quando comparadas às adultas (ver Tabela 2).

Quanto à VS, o estupro foi mais frequentemente notificado, seguido da exploração sexual e do assédio sexual. Na maioria dos casos ocorreu penetração vaginal e outras práticas sexuais, como sexo oral e anal. Nessas situações, verificou-se a prevalência da violência sem o uso do preservativo. Os agressores usaram a força corporal e espancamento e ameaçaram as vítimas, utilizando-se de enforcamento, objetos contundente ou cortante e armas de fogo, além de outros tipos de agressão. O local de ocorrência da violência mais relatado foi a residência, seguido da via pública, terreno baldio e bar.

Em relação ao agressor, verificou-se predominância de agressor do sexo masculino na totalidade dos casos notificados. Além disso, o agressor fez uso 


\section{Tabela 1}

Descrição da violência, agressor, atendimento, consequências da violência e desfecho da gestação.

\begin{tabular}{|c|c|}
\hline & $\mathrm{f}(\%)$ \\
\hline \multicolumn{2}{|l|}{ Caracterização da violência } \\
\hline Sexual & $112(100 \%)$ \\
\hline Física & $36(46,1 \%)$ \\
\hline Psicológica/Moral & $34(43,6 \%)$ \\
\hline Tortura & $4(5,1 \%)$ \\
\hline Financeira & $2(2,6 \%)$ \\
\hline Negligência & $2(2,6 \%)$ \\
\hline \multicolumn{2}{|l|}{ Tipo de violência sexual } \\
\hline Estupro & $92(95,8 \%)$ \\
\hline Exploração sexual & $3(3,1 \%)$ \\
\hline Assédio sexual & $1(1,0 \%)$ \\
\hline Violência extrafamiliar & $71(75,5 \%)$ \\
\hline Violência intrafamiliar & $23(24,5 \%)$ \\
\hline \multicolumn{2}{|l|}{ Práticas sexuais } \\
\hline Penetração vaginal & $69(80,2 \%)$ \\
\hline Oral e anal & $15(17,4 \%)$ \\
\hline Anal & $2(1,8 \%)$ \\
\hline Não usou preservativo & $64(73,6 \%)$ \\
\hline \multicolumn{2}{|l|}{ Outras agressões } \\
\hline Força corporal/Espancamento & $51(60,0 \%)$ \\
\hline Ameaças & $18(21,8 \%)$ \\
\hline Outros & $16(18,8 \%)$ \\
\hline \multicolumn{2}{|l|}{ Local da ocorrência } \\
\hline Residência & $38(33,9 \%)$ \\
\hline Via pública & $22(19,6 \%)$ \\
\hline Terreno baldio & $13(11,6 \%)$ \\
\hline Bar & $4(3,6 \%)$ \\
\hline Outros & $19(17,0 \%)$ \\
\hline \multicolumn{2}{|l|}{ Agressor } \\
\hline Quantidade (1 agressor) & $75(80,6 \%)$ \\
\hline \%Masculino & $112(100 \%)$ \\
\hline Usou drogas & $41(60,3 \%)$ \\
\hline \multicolumn{2}{|l|}{ Vínculo com agressor } \\
\hline Desconhecido & $46(40,4 \%)$ \\
\hline Amigo/Conhecido & $19(16,7 \%)$ \\
\hline Cônjuge/Namorado & $12(10,6 \%)$ \\
\hline Padrasto & $4(3,5 \%)$ \\
\hline Pai biológico & $3(2,6 \%)$ \\
\hline Irmão & $2(1,8 \%)$ \\
\hline Relação institucional & $1(0,9 \%)$ \\
\hline Outros & $5(4,4 \%)$ \\
\hline Notificação (BO) & $59(64,8 \%)$ \\
\hline
\end{tabular}




\begin{tabular}{lc} 
Continuação & \\
\hline Início do atendimento hospitalar & $51(59,3 \%)$ \\
72 horas & $19(22,1 \%)$ \\
1 mês & $16(18,6 \%)$ \\
2 meses ou mais & \\
Encaminhamentos & $21(18,8 \%)$ \\
Ambulatorial & $20(17,9 \%)$ \\
Hospitalar & $71(63,4 \%)$ \\
Informação ausente & $62(55,4 \%)$ \\
Outros setores & \\
Consequências da violência & $37(31,1 \%)$ \\
Gravidez & $2(1,8 \%)$ \\
DST & $7(6,3 \%)$ \\
Estresse pós-traumático & $3(2,7 \%)$ \\
Outros & \\
Desfecho da gestação & $5(6,3 \%)$ \\
Gestou & $12(15,2 \%)$ \\
Interrompeu & $20(25,3 \%)$ \\
Informação ausente &
\end{tabular}

BO: Boletim de Ocorrência; DST: Doença Sexualmente Transmissível.

de álcool e outras drogas. Ressalta-se que as adolescentes quando comparadas às adultas foram violentadas com maior frequência por mais de um agressor $(\mathrm{t}=1,8 ; \mathrm{df}=70 ; \mathrm{p}<0,001$; ver Tabela 2$)$. A VS foi caracterizada como extrafamiliar na maioria dos casos analisados, sendo os agressores identificados como desconhecidos, seguido de amigos/conhecidos, pessoa com relação institucional, além de outros. Nas violências intrafamiliares, os agressores foram cônjuge/namorado, padrasto, pai biológico e irmão. Quando se comparou as vítimas adolescentes e adultas no que se refere à caracterização da violência como intrafamiliar ou extrafamiliar (Tabela 2), verificou-se que as adolescentes foram mais frequentemente vítimas da violência intrafamiliar do que as adultas $(\mathrm{t}=2,7 ; \mathrm{df}=77 ; \mathrm{p}<0,001)$.

\section{Atendimento recebido, consequências da violência e desfecho da gestação}

A VS sofrida foi revelada em outros contextos além do hospital, sendo a delegacia o contexto mais buscado para denúncia da violência por meio do boletim de ocorrência. A maioria das vítimas buscou atendimento no hospital em 72 horas. Verificou-se que as adolescentes chegaram com maior rapidez ao hospital do que as adultas $(\mathrm{t}=-1,9 ; \mathrm{df}=83,8 ; \mathrm{p}<0,01$; ver Tabela 2). Após iniciarem atendimento no hospital, as vítimas receberam encaminhamento ambulatorial e hospitalar. Essa informação esteve ausente em grande parte dos casos. As dificuldades de continuidade do atendimento também foram observadas nas informações sobre a evolução do tratamento, visto que apenas três formulários identificaram alta das vítimas, os demais não apresentaram quaisquer informações. Além do atendimento no hospital, as vítimas receberam encaminhamentos para outros setores, tais como conselho tutelar, delegacia de proteção da criança e do adolescente e centro de referência da mulher.

Em relação às consequências daVS, identificou-se gravidez, DSTs, estresse pós-traumático, entre outros. Entre as que engravidaram, verificou-se que um maior número de mulheres interrompeu $(\mathrm{n}=12 ; 15,2 \%)$ a gestação do que ao daquelas que deram continuidade $(n=5 ; 6,3 \%)$.Verificaram-se, ainda, casos de mulheres que abandonaram o atendimento hospitalar, não se identificando informações sobre sua situação gestacional. Salienta-se também que em muitos dos casos em que as mulheres engravidaram em decorrência da violência sexual, a informação sobre o desfecho da 
gestação não esteve presente nos formulários. Ressalta-se que, entre as mulheres que engravidaram, as adultas interromperam a gestação mais frequentemente do que as adolescentes $(\mathrm{t}=-2,09 ; \mathrm{df}=8,9 ; \mathrm{p}<$ 0,01; ver Tabela 2).

\section{Correlações entre variáveis relacionadas à vítima, violência, agressores e desfecho da gestação}

Conforme mostra a Tabela 3, análises de correlação verificaram que a faixa etária teve correlação negativa com violência intrafamiliar, indicando que as adolescentes sofreram mais frequentemente violência intrafamiliar. A violência intrafamiliar teve correlação positiva com número de violência sofrida, sugerindo que as mulheres que foram vítimas de violência intrafamiliar afirmaram ter sofrido mais frequentemente violência física, psicológica/moral, tortura, econômica e/ou negligência, além da violência sexual.

Em relação à gravidez decorrente do estupro, as correlações mostraram que as mulheres que sofreram violência intrafamiliar engravidaram mais e aquelas que foram violentadas por mais de um agressor não engravidaram. Quanto ao início do

\section{Tabela 2}

Comparações entre faixa etária quanto às variáveis de caracterização da violência (número e tipo), quantidade de agressores, consequência da violência (gravidez) e desfecho da gestação.

\begin{tabular}{lcc}
\hline Variável & Adolescentes & Adultas \\
\hline & $\mathrm{M}(\mathrm{DP})$ & $\mathrm{M}(\mathrm{DP})$ \\
Número de violência (1-6) & $1,75(0,84)$ & $1,68(0,79)$ \\
Violência intrafamiliar (0-1) & $0,39(0,49)^{* *}$ & $0,14(0,35)^{* *}$ \\
Quantidade de agressores (1-2) & $1,28(0,45)^{* *}$ & $1,13(0,34)^{* *}$ \\
Início do atendimento (0-2) & $0,42(0,65)^{*}$ & $0,90(1,16)^{*}$ \\
Consequência/gravidez (0-1) & $0,40(0,5)$ & $0,53(0,5)$ \\
Desfecho da gestação $(1-2)$ & $1,43(0,53)^{*}$ & $1,9(1,0)^{*}$ \\
\hline
\end{tabular}

Número de violência: 1 - um tipo de violência a 6 - todos os tipos de violência;

Violência intrafamiliar: 0 - violência extrafamiliar a 1 - violência intrafamiliar;

Quantidade de agressores: 1 - um agressor a 2 - dois ou mais agressores;

Início do atendimento: 0 - 72 horas a 2 - dois meses ou mais;

Consequência/gravidez: 0 - não engravidou a 1 - engravidou;

Desfecho da gestação: 1 - continuidade da gestação a 2 - interrompeu a gestação.

${ }^{*} \mathrm{p}<0,01{ }^{* *} \mathrm{p}<0,001$

\section{Tabela 3}

Correlações entre faixa etária, caracterização da violência (número e tipo), quantidade de agressores, consequência da violência (gravidez) e desfecho da gestação.

\begin{tabular}{lccccccc}
\hline & 1 & 2 & 3 & 4 & 5 & 6 & 7 \\
\hline Faixa etária $^{1}$ & - & $-0,04$ & $-0,28^{* *}$ & $0,24^{*}$ & $-0,18$ & 0,13 & $0,55^{* *}$ \\
Número de violências $^{2}$ & - & - & $0,24^{*}$ & $-0,1$ & 0,05 & $-0,09$ & $-0,19$ \\
Violência intrafamiliar $^{3}$ & - & - & - & $-0,07$ & $-0,14$ & $0,25^{*}$ & $-0,53^{*}$ \\
Início do atendimento $^{4}$ & - & - & - & - & $-0,02$ & $0,79^{* * *}$ & 0,14 \\
Quantidade de agressores $^{5}$ & - & - & - & - & - & $-0,33^{* *}$ & 0,15 \\
Consequência/gravidez $^{6}$ & - & - & - & - & - & - & 0,17 \\
Desfecho da gestação $^{7}$ & - & - & - & - & - & -- & - \\
\hline
\end{tabular}

Faixa etária: adolescente $=1$; adulta $=2$.

${ }^{*} \mathrm{p}<0,05,{ }^{* *} \mathrm{p}<0,01,{ }^{* * *} \mathrm{p}<0,001$. 
atendimento, os achados das correlações apontaram que as mulheres que chegaram mais tardiamente ao serviço de saúde eram adultas e tinham engravidado. Entre as mulheres que engravidaram, as adultas mais frequentemente escolheram interromper a gestação e as vítimas de violência intrafamiliar deram continuidade à gestação.

\section{Discussão}

Acerca das vítimas de VS, estudos nacionais e internacionais apontam ser o sexo feminino o mais suscetível à violência sexual e os homens os principais autores de agressão. Isso explica-se principalmente pela relação desigual de poder, em razão da opressão de gênero existente entre o binômio homem-mulher. Trata-se de práticas discriminatórias construídas ao longo do tempo e que se apresentam de diversas formas, sendo uma delas através da dominação dos corpos das mulheres, que as tornam mais suscetíveis a vivenciarem relações violentas (Narvaz, \& Koller, 2006).

Também que entre as mulheres destacam-se características como: ser jovem, de raça/cor parda, solteira, estudante e de nível escolar inferior (Andrade et al., 2001; Gomes et al., 2006; Justino et al., 2015), como verificou-se nos achados deste estudo. No entanto, é importante ressaltar, apesar de não ser a ênfase deste trabalho, que a VS não se restringe ao sexo masculino como agressor e que também os tornam vítimas, sendo principalmente crianças e adolescentes. Tal problemática ainda é pouco notificada e uma minoria dos casos chega aos serviços de saúde por questões relacionadas a medo, vergonha e estereótipos associados (Justino et al., 2015).

Dentre as formas de VS, o estupro foi a forma de violência mais notificada, compreendendo que com a abrangência do crime de estupro, de modo a englobar o antigo crime de Atentado Violento ao Pudor (AVP) (Lei no 12.015/2009), o crime de estupro passou a considerar também outras práticas libidinosas e não somente as que envolviam a "conjunção carnal” (Brasil, 2009). Deste modo, a análise feita por este estudo já contempla a reformulação da lei, apesar de que se observou que a ficha de notificação da violência utilizada pelo serviço hospitalar ainda datava de época anterior às alterações na lei, em que se apresentava como opção o item de AVP. Este fato destaca que tanto a gestão do hospital como os profissionais responsáveis pelo preenchimento das fichas estavam alheios a essa mudança.

Sobre o autor da VS, predominantemente do sexo masculino neste estudo, destacou-se por ser uma pessoa desconhecida por parte das vítimas, tendo agido sozinho, sob suspeita de uso de álcool ou outra droga e tendo utilizado como recurso para a agressão: força corporal, espancamento, ameaças, objetos cortantes, armas de fogo, entre outros. Segundo Cerqueira e Coelho (2014), o indivíduo desconhecido passa a configurar paulatinamente como principal autor do estupro à medida que a idade da vítima aumenta, o que justifica a diferença de vínculo entre o agressor e mulheres adolescentes e adultas. Facuri et al. (2013) destacaram este aspecto, apontando que a violência por agressor conhecido (frequentemente intrafamiliar) está mais presente em mulheres mais jovens, além de menor taxa de procura por auxílio e maior número de atos praticados. Já em relação às mulheres adultas, a violência é mais frequentemente praticada por agressor desconhecido e são maiores as taxas de agressão física, de denúncia e de procura por auxílio.

A violência que predominou contra vítimas adolescentes foi a do tipo intrafamiliar em contraponto às mulheres adultas, corroborando os achados de outros estudos (Facuri et al., 2013; Justino et al., 2015), que apontam estarem as adolescentes mais suscetíveis à violência dentro do lar e por entes próximos. Observou-se que é mais frequente entre as adolescentes a coexistência de mais de um tipo de violência, como física, sexual e psicológica. De acordo com a literatura, quando a violência é do tipo intrafamiliar, maior pode ser o comprometimento à saúde das vítimas, pelo vínculo estabelecido com o agressor, pela frequência das agressões, pela intensidade do fato percebido pela vítima e pela maturidade para compreendê-lo (Habigzang, \& Koller, 2006; Williams, 2002). Tais fatores podem justificar a maior diversidade de violência quando a vítima é adolescente, pela convivência que esta possui com o agressor.

Quanto às consequências da violência sexual, confirmaram-se os problemas de saúde já apontados em outros estudos, como estresse pós-traumático, transtorno comportamental e gravidez (Justino et al., 2015), apesar de maior incidência da gravidez como consequência entre as vítimas. Tal fato pode estar associado ao não uso de preser- 
vativo por parte do agressor, assim como à demora quanto à chegada ao serviço de saúde, que, após as $72 \mathrm{~h}$ da ocorrência da violência, inviabiliza a administração da anticoncepção de emergência. Essa realidade permite refletir sobre o conhecimento dessas mulheres acerca dos serviços de saúde que prestam cuidados em situação de VS, bem como sobre a divulgação desses serviços e das questões emocionais, culturais, sociais que possam interferir nessa chegada ao serviço de saúde (Bedone, \& Faúndes, 2007; Mattar et al., 2007; Oliveira et al., 2005; Silva, Billings, Garcia, \& Lara, 2009; Villela, \& Lago, 2007). Outra questão que pode estar associada é a forma de violência, visto que, quando a vítima convive com o agressor (violência intrafamiliar), mais suscetível ela pode estar a engravidar pela frequência com que ocorre a VS (Cerqueira, \& Coelho, 2014).

Quando diagnosticada a gravidez, as adolescentes tenderam mais frequentemente a dar continuidade à gestação. Estudos apontaram que a interrupção da gestação se reduz quando a grávida é adolescente ou criança. Um dos motivos diz respeito à exigência da norma técnica do Ministério da Saúde (Brasil, 2012) que prescreve que o aborto para menores de 18 anos só deve ser feito quando a criança/adolescente e o seu responsável concordam. Nos casos de interrupção da gravidez em adolescentes, a família necessariamente é comunicada acerca da violência e da gestação, visto que a jovem não pode por si só requerer a interrupção da gestação sem a presença e assinatura de um responsável, ao contrário das mulheres adultas. Nesse sentido, a interferência da família no processo pode ser um fator a favor da continuidade da gestação. Outro motivo deve-se à alta prevalência de casos de estupro envolvendo crianças e adolescentes, nos quais os próprios familiares são os autores, sendo possível que a diferença na taxa de aborto entre menores de idade e adultos reflita esses fatos (Cerqueira, \& Coelho, 2014).

Acerca dos encaminhamentos das vítimas dentro do próprio hospital e a serviços externos a este, os dados mostraram-se frágeis, apesar de terem sido identificados encaminhamentos ambulatoriais e de internação, bem como para outros serviços tais como conselho tutelar, delegacia de proteção da criança e do adolescente e centro de referência da mulher. É importante destacar que a continuidade do atendimento à vítima e sua família, quando presente no caso, se torna essencial para minimizar os danos provocados pela ocorrência da violência, restaurar planos anteriores e excitar projetos futuros. Entre os serviços de proteção às vítimas e suas famílias estão os serviços de saúde, da assistência social, da educação, de justiça, de assistência social, educação, justiça, segurança pública, Ministério Público, Defensoria Pública, Varas da Infância e Juventude, Conselho Tutelar, Varas de Violência Doméstica e Famíliar contra a mulher, entre outros, que devem ser utilizados pelo tempo que necessitarem (Brasil, 2010; 2012).

Por fim, observou-se que muitas das informações pertinentes ao preenchimento das fichas analisadas estiveram ausentes, incompletas ou incorretas, invalidando o item analisado. Portanto, verificou-se a fragilidade profissional quanto ao manejo das fichas, percebida em deficiência de conhecimento da temática, de responsabilidade para com o preenchimento e de disposição para escuta atenta e qualificada que garantam a qualidade das informações. Alguns estudos ressaltaram essa problemática, levantando a qualidade da informação das notificações, visto o nível elevado de ausência de registros em alguns campos da ficha (Assis, Avanci, Pesce, Pires, \& Gomes, 2012; Cerqueira, \& Coelho, 2014). $\mathrm{O}$ ato de notificar é um exercício de cidadania, que leva ao cuidado e à proteção da vítima. Também possibilita o conhecimento do perfil da violência para intervenção, prevenção, planejamento e avaliação de políticas públicas (Veloso et al., 2013). Por essas razões, considera-se importante a necessidade da sensibilização e treinamento dos profissionais para o preenchimento correto das fichas, fato que certamente trará benefícios para a rede de atendimento como um todo, principalmente para as vítimas de violência sexual (Adriasola, 2013; Assis et al., 2012; De Zordo, 2012; Duarte, \& Almeida, 2010; Farias, \& Cavalcanti, 2012).

\section{Considerações finais}

Este estudo teve como objetivo descrever as características da vítima, da violência, do agressor e do atendimento recebido por mulheres ( 12 anos acima) em um hospital da rede pública de Fortaleza-CE entre 2010 e 2013. Buscou-se, ainda, identificar as consequências da violência e o desfecho da gestação (continuidade ou interrupção), além de realizar uma análise comparativa das vítimas adolescentes e adultas.

As vítimas destacaram-se por serem em sua maioria adultas, de raça/cor parda, heterossexual, 
solteira e estudante. A violência sexual mais praticada foi o estupro e por agressor desconhecido por parte das vítimas. Contudo, quando a agressão era cometida contra as adolescentes, percebeu-se que predominava a violência do tipo intrafamiliar e com a presença de mais de uma forma de violência. Acerca das consequências da violência sexual, a gravidez apareceu como a mais frequente, tendo as mulheres adolescentes mais vezes optado pela continuidade da gestação, enquanto as mulheres adultas mais vezes optaram pela interrupção. Sobre a evolução do atendimento, em muitos casos não foi possível saber sobre a continuidade do acompanhamento, devido à fragilidade de preenchimento das fichas analisadas, muitas delas com informações ausentes, incompletas ou incorretas.

A fim de que a assistência às vítimas seja realmente completa e de qualidade faz-se necessário o fortalecimento da rede de proteção, constituída de maneira interdisciplinar e intersetorial, inclusive com participação da comunidade. Além de políticas que favoreçam a capacitação profissional para a identificação, notificação, tratamento adequado, acompanhamento dos casos e encaminhamentos, proporcionando a implementação de estratégias eficazes para a prevenção de novos casos e minimização das consequências de casos notificados. Enfatiza-se a necessidade de aprimorar a formação acadêmica sobre a temática estudada em todos os cursos da saúde e ciências humanas e sociais, para melhor compreensão do fenômeno em sua dinâmica relacional (Justino et al., 2015).

Destaca-se a importância da continuidade de pesquisas nesta área, tais como estudos longitudinais que investiguem a situação de vida das vítimas adolescentes e adultas após a ocorrência da violência e a passagem pelo serviço de saúde; estudos de caso que descrevam o percurso da vítima nos serviços de proteção; estudos acerca das notificações das violências, entre outros. Em relação ao último aspecto, encontra-se a limitação deste estudo, visto a grande quantidade de informações ausentes nas fichas analisadas, que impossibilitaram a realização de outras análises, além das que foram apresentadas neste trabalho. Lamentavelmente, ainda há resistência do profissional para preencher mais um instrumento com grande número de variáveis e medo de represálias do autor da violência ao notificar. No entanto, em termos de contribuições deste estudo, está a variedade de informações acerca da população vítima de VS, especificamente do grupo de mulheres, adolescentes e adultas, que possibilita informar sobre diversas perspectivas acerca da VS (da vítima, da violência, do agressor, do atendimento) e, com isso, subsidiar o planejamento de políticas públicas voltadas para esse público.

\section{Referências}

Adriasola, G. (2013). La objeción de conciencia y la interrupción voluntaria Del embarazo: cómo conciliar su ejercicio con los derechos de lãs usuarias? Revista Médica del Uruguay, 29(1), 47-57.

Andrade, R. P., Guimarães, A. C. P., Fagotti Filho, A., Arrabal, N. S. C. J. S., Rocha, D. M., \& Medeiros, J. M. (2001). Características demográficas e intervalo para atendimento em mulheres vítimas de violência sexual. Revista Brasileira de Ginecologia Obstetrícia, 23(9), 583-587. https://doi.org/10.1590/S010072032001000900006

Assis, S. G., Avanci, J. Q., Pesce, R. P., Pires, T. O., \& Gomes, D. L. (2012). Notificações de violência doméstica, sexual e outras violências contra crianças no Brasil. Ciência \& Saúde Coletiva, 17(9), 2305-2317. https://doi.org/10.1590/ S1413-81232012000900012

Bedone, A. J., \& Faundes, A. (2007). Atendimento integral às mulheres vítimas de violência sexual: Centro de Assistência Integral à Saúde da Mulher, Universidade Estadual de Campinas. Cadernos de Saúde Pública, 23(2), 465-469. https://doi.org/10.1590/S0102-311X2007000200024

Brasil. (2009, 10 de agosto). Lei No 12.015, de 7 de agosto de 2009. Altera o Título VI da Parte Especial do Decreto-Lei no 2.848, de 7 de dezembro de 1940 - Código Penal, e o art. 1 o da Lei no 8.072, de 25 de julho de 1990, que dispõe sobre os crimes hediondos, nos termos do inciso XLIII do art. 5o da Constituição Federal e revoga a Lei no 2.252, de 1 o de julho de 1954, que trata de corrupção de menores. Diário Oficial da União. 
Brasil. (1990, 16 de julho). Lei No 8.069, de 13 de julho de 1990. Dispõe sobre o Estatuto da Criança e do Adolescente e dá outras providências. Diário Oficial da União.

Brasil. (2010). Ministério da Saúde. Secretaria de Atenção à Saúde. Departamento de Ações Programáticas Estratégicas. Linha de cuidado para a atenção integral à saúde de crianças, adolescentes e suas famílias em situação de violências: orientação para gestores e profissionais de saúde (Série F. Comunicação e Educação em Saúde). Brasília, DF: Ministério da Saúde.

Brasil.. (2012). Ministério da Saúde Secretaria de Atenção à Saúde. Departamento de Ações Programáticas Estratégicas. Prevenção e tratamento dos agravos resultantes da violência sexual contra mulheres e adolescentes: norma técnica (3a ed.). Brasília, DF: Ministério da Saúde.

Cantelmo, C., Cavalcante, T., \& Costa, L. (2011). A menina mãe: incesto e maternidade. Fractal: Revista de Psicologia, 23(1), 137-154. https://doi.org/10.1590/S1984-02922011000100010

Cerqueira, D., \& Coelho, D. S. C. (2014). Estupro no Brasil: uma radiografia segundo os dados da Saúde. Brasília, DF: IPEA.

De Zordo, S. (2012). Representações e experiências sobre aborto legal e ilegal dos ginecologistas-obstetras trabalhando em dois hospitais maternidade de Salvador da Bahia. Ciências \& Saúde Coletiva, 17(7), 1745-1754. https://doi.org/10.1590/S1413-81232012000700013

Drezett, J., Pedroso, D., Gebrim, L. H., Matias, M. L., Macedo Júnior, H., \& Abreu, L. C. (2011). Motivos para interromper legalmente a gravidez decorrente de estupro e efeitos do abortamento nos relacionamentos cotidianos das mulheres. Reprodução \& Climatério, 26(3), 85-91.

Duarte, D. A., \& Almeida, M. G. M. (2010). Conhecimento dos profissionais de saúde frente ao aborto legal no brasil: uma revisão bibliográfica. Revista Baiana de Saúde Pública, 34(2), 279-287.

Facuri, C. O., Fernandes, A. M. S., Oliveira, K. D, Andrade, T. S., \& Azevedo, R. C. S. (2013). Violência sexual: estudo descritivo sobre as vítimas e o atendimento em um serviço universitário de referência no Estado de São Paulo, Brasil. Cadernos de Saúde Pública, 29(5), 889-898. https://doi.org/10.1590/S0102-311X2013000500008

Farias, R. S., \& Cavalcanti, L. F. (2012). Atuação diante das situações de aborto legal na perspectiva dos profissionais de saúde do Hospital Municipal Fernando Magalhães. Ciências \& Saúde Coletiva, 17(7), 1755-1763. https://doi.org/10.1590/S1413-81232012000700014

Faúndes, A., Rosas, C. F., Bedone, A. J.,\& Orozco, L. T. (2006). Violência sexual: procedimentos indicados e seus resultados no atendimento de urgência de mulheres vítimas de estupro. Revista Brasileira de Ginecologia e Obstetrícia, 28(2), 126-135. https://doi.org/10.1590/S0100-72032006000200009

Gomes, M. L. M., Falbo Neto, G.H., Viana, C. H., \& Silva, M. A. (2006). Perfil clínico-epidemiológico de crianças e adolescentes do sexo feminino vítimas de violência atendidas em um Serviço de Apoio à Mulher, Recife, Pernambuco. Revista Brasileira de Saúde Materno Infantil, 6(suppl 1), S27-34. https://doi.org/10.1590/S151938292006000500004

González, E., Luengo, X., Caba, F., \& Molina, T. (1999). El contexto sociofamiliar de loshijos de madres adolescentes cuyos embarazos fueron consecuencia de violación. Cuadernos Médicos Sociales (Santiago de Chile), $40(2), 25-32$.

Habigzang, L. F., \& Koller, S. H. (2006). Terapia cognitivo-comportamental e promoção de resiliência para meninas vítimas de abuso sexual intrafamiliar. In D. D. Dell'Aglio, S. H. Koller, \& M. A. M. Yunes (Eds.), Resiliência e psicologia positiva: Interfaces do risco à proteção (pp. 233-258). São Paulo, SP: Casa do Psicólogo.

Justino, L. C. L., Nunes, C. B., Gerk, M. A. S., Fonseca, S. S. O., Ribeiro, A. A., \& Filho, A. C. P. (2015). Violência sexual contra adolescentes em Campo Grande, Mato Grosso do Sul. Revista Gaúcha de Enfermagen, 36(no. esp), 239246. https://doi.org/10.1590/1983-1447.2015.esp.56820

Lima, C. A., \& Deslandes, S. F. (2014). Violência sexual contra mulheres no Brasil: conquistas e desafios do setor saúde na década de 2000. Saúde e Sociedade, 23(3), 787-800. https://doi.org/10.1590/S010412902014000300005

Machado, C. L., Fernandes, A. M. S., Osis, M. J. D., \& Makuch, M. Y. (2015). Gravidez após violência sexual: vivências de mulheres em busca da interrupção legal. Cadernos de Saúde Pública, 31(2), 345-353. https:// doi.org/10.1590/0102-311X00051714 
Mattar, R., Abrahão, A. R., Andalaft Neto, J., Colas, O. R., Schroeder, I., Machado, S. J. et. al. (2007). Assistência multiprofissional à vítima de violência sexual: a experiência da Universidade Federal de São Paulo. Caderno de Saúde Pública, 23(2), 459-464. https://doi.org/10.1590/S0102-311X2007000200023

Matos, M. (2008). Teorias de gênero ou teorias e gênero? Se e como os estudos de gênero e feministas se transformaram em um campo novo para as ciências. Estudos Feministas, 16(2), 333-357. https://doi.org/10.1590/S0104026X2008000200003

Moura, E. R. F; Silveira, L. C., \& Nogueira, R. A. (2001). A realidade do abuso sexual na vida de uma adolescente: relato de caso. Revista da Rede de Enfermagem do Nordeste, 2(1), 15-19. https://doi.org/10.15253/rev\%20rene. v2i1.5798

Narvaz, M. G., \& Koller, S. H. (2006). Metodologias feministas e estudos de gênero: articulando pesquisa, clínica e política. Psicologia em Estudo, 11(3), 647-654. https://doi.org/10.1590/S1413-73722006000300021

Nunes, M. C. A., \& Morais, N. A. (2016). Violência sexual e gravidez: percepções e sentimentos das vítimas. Revista da SPAGESP, 17(2), 21-36.

Oliveira, E. M., Barbosa, R. M., Moura, A. A. V. M., Kossel, K., Morelli, K., Botelho, L. F. F. et. al. (2005). Atendimento às mulheres vítimas de violência sexual: um estudo qualitativo. Revista de Saúde Pública, 39(3), 376-382. https:// doi.org/10.1590/S0034-89102005000300007

Santos, A. P. (2012). Violência sexual infantojuvenil: problematizando os desafios do atendimento a partir da análise de um caso clínico. Mental, 10(18), 89-108.

Silva, M., Billings, D. L., García, S. G., \& Lara, D. (2009). Physicians' agreement with and willingness to provide abortion services in the case of pregnancy from rape in Mexico. Contraception, 79(1), 56-64. https://doi.org/10.1016/j. contraception.2008.07.016

Veloso, M. M. X., Magalhães, C. M. C., Dell'Aglio, D. D., Cabral, I. R., \& Gomes, M. M. (2013). Notificação da violência como estratégia de vigilância em saúde: perfil de uma metrópole do Brasil. Ciência e Saúde Coletiva, 18(5), 12631272. https:// doi.org/10.1590/S1413-81232013000500011

Vertamatti, M. A. F., Souza, J. V. L., Vieira, S., Ohata, A. P., Sancovski, M., Abreu, L. C., \& Barbosa, C. P. (2009). Parto cesárea em gravidez decorrente de estupro. Revista Brasileira de Crescimento e Desenvolvimento Humano, 19(2), 327-331. https://doi.org/10.7322/jhgd.19922

Villela, W. V., \& Lago, T. (2007). Conquistas e desafios no atendimento das mulheres que sofreram violência sexual. Cadernos de Saúde Pública, 23(2), 471-475. https://doi.org/10.1590/S0102-311X2007000200025

Williams, L. C. A. (2002). Abuso sexual infantil. In H. J. Guilhardi, M. B. B. Madi, P. P. Queiroz, \& M. C. Scoz (Orgs.), Sobre comportamento e cognição: contribuições para a construção da teoria do comportamento. Santo André, SP: ESETec.

World Health Organization - WHO. (2002). World report on violence and health. Geneva: World Health Organization.

Mykaella Cristina Antunes Nunes

Doutoranda em Psicologia, Universidade de Fortaleza (UNIFOR)

Rebeca Fernandes Ferreira Lima

Doutoranda em Psicologia, Universidade de Fortaleza (UNIFOR)

Normanda Araujo de Morais

Doutora em Psicologia, Universidade de Fortaleza (UNIFOR)

Endereço para envio de correspondência:

Av. Washington Soares, 1321, Edson Queiroz, 60811-905, Fortaleza, CE, Brasil. 
Recebido 29/08/2016

Reformulado 28/03/2017

Aprovado 03/08/2017

Received $08 / 29 / 2016$

Reformulated $03 / 28 / 2017$

Approved 08/03/2017

Recibido 29/08/2016

Reformulado 28/03/2017

Aceptado 03/08/2017

Como citar: Nunes, M. C. A., Lima, R. F. F., Morais, N. A.Violência Sexual contra Mulheres: um Estudo Comparativo entre Vítimas Adolescentes e Adultas. Psicologia: Ciência e Profissão, 37(4), 956-969. https://doi.org/10.1590/1982-3703003312016

How to cite: Nunes, M. C. A., Lima, R. F. F., Morais, N. A.Sexual violence against women: a comparative study between adolescent and adult victims. Psicologia: Ciência e Profissão, 37(4), 956-969. https://doi.org/10.1590/1982-3703003312016

Cómo citar: Nunes, M. C. A., Lima, R. F. F., Morais, N. A.Violencia Sexual contra mujeres: un Estudio Comparativo entre Víctimas Adolescentes y Adultas. Psicologia: Ciência e Profissão, 37(4), 956-969. https://doi.org/10.1590/1982-3703003312016 